\title{
Development of beetroot (Beta vulgaris) powder using foam mat drying
}

\begin{abstract}
Beetroot (Beta vulgaris) is normally used as natural food colorants in food industry. The objectives of this research are (1) to determine the effect of types (egg albumen and fish gelatine) and different concentrations of foaming agents on foam properties of foam mat drying beetroot, (2) to evaluate the physicochemical properties (color, $\mathrm{pH}$, Brix, bulk density, water activity, hygroscopicity and moisture content) of foam dried beetroot powder, and (3) to determine the effect of temperature on rehydration ratio and color changes of rehydrated foam mat dried beetroot powder. Beetroot foam was spread at $3 \mathrm{~mm}$ thickness and hot air dried at $50{ }^{\circ} \mathrm{C}$ for $6 \mathrm{~h}$ in cabinet dryer. Beetroot pulp can be successfully foamed using egg albumen and fish gelatine as foaming agents, further hot air dried and grinded into powder. In this experiment, beetroot powder after foam mat drying was considered as hygroscopic food and resulted in lighter and reddish in colour. After rehydrating foam mat dried beetroot powder, $L^{*}$ value decreased but $a^{*}$ and $b^{*}$ values increased. Generally, sample beetroot with fish gelatine (BFG) had good foam expansion, foam density, hygroscopicity, water activity, and red color's powder.
\end{abstract}

Keyword: Foam mat drying; Beetroot; Fish gelatin; Egg albumen; Natural colorant 